\title{
The relation between plasma miR-126 levels and cerebral collateral circulation in patients with intracranial arterial stenosis
}

\author{
Xiwa Hao ${ }^{1,2,3 \star}$, Shuwan Wang ${ }^{1,3 \star}$, Changchun Jiang ${ }^{1,2,3}$, Jingfen Zhang ${ }^{1,2,3}$, Yu Fan $^{1,2,3}$, Jiangxia Pang ${ }^{1,2}$, \\ Tianyou Zhang ${ }^{1,3}$, Fei Hao ${ }^{1}$, Junfeng Yang ${ }^{1,2}$, Xia Li ${ }^{1,2}$, Jiahui Liu ${ }^{1,2}$, Baojun Wang ${ }^{1,2,3}$, Yuechun Li ${ }^{1,2,3}$ \\ ${ }^{1}$ Department of Neurology, Baotou Central Hospital, Inner Mongolia, China \\ ${ }^{2}$ Institute of Cerebrovascular Disease in Inner Mongolia, Inner Mongolia, China \\ ${ }^{3}$ Clinical Research Centre for Neurological Diseases in Inner Mongolia Autonomous Region, Inner Mongolia, China
}

\begin{abstract}
Objective. This study aimed to investigate the correlation between the circulating miR-126 regulation pathway and the cerebral collateral circulation (CCC), and to test whether miR-126 could serve as a potential biomarker for CCC formation in patients with intracranial arterial stenosis or occlusion.

Material and methods. This single-centre cross-sectional study enrolled patients who underwent cerebral angiography with severe stenosis $(\geq 70 \%)$ or occlusion in at least one major intracranial artery. Collateral degree was graded according to the ASITN/SIR classification. The patients were divided into a good CCC group (grade 3-4) or a poor CCC group (grade 0-2). We investigated the plasma levels of miR-126, VEGF, Spred-1 and PIK3R2 by using qRT-PCR, ELISA and Western blot methods, respectively. In addition, we assessed the correlations of plasma miR-126 with VEGF, Spred-1, PIK3R2 and ASITN/SIR grade using the Spearman correlation test and investigated its predictive power for $\mathrm{CCC}$ status by using the receiver operating characteristic curve.

Results. A total of 68 patients were enrolled (44 with good CCC and 24 with poor (CC). Data showed that plasma miR-126 and VEGF were significantly higher in the good $C C C$ group than in the poor $C C C$ group. Plasma Spred-1 and PIK3R2 level were lower in the good CCC group than in the poor CCC group. In addition, miR-126 and VEGF were positively correlated with ASITN/SIR ( $m i R-126: R=0.595, P<0.01 ; V E G F: R=0.595, P<0.01$ ), whereas Spred-1 and PIK3R2 were negatively correlated with ASITN/SIR (Spred-1: $R=-0.817, P<0.01 ; P I K 3 R 2: R=-0.513, P=0.01$ ). However, the area under the curve of miR-126 level for $C C C$ status was only 0.328 (95\% Cl: $0.158-0.498 ; p=0.067)$.
\end{abstract}

Conclusions. Plasma miR-126 level may be related to better CCC formation, one of the mechanisms that may be explained by upregulation of VEGF and reduction of Spred-1 and PIK3R2 protein expression. However, miR-126 might not be an independent predictor for CCC, given its low predictive value.

Key words: miR-126, VEGF, cerebral collateral circulation, ASITN/SIR, biomarker

(Neurol Neurochir Pol 2021; 55 (3): 281-288)

\section{Introduction}

Acute ischaemic stroke can result in severe neurological disability and/or death [1]. The cerebral collateral circulation (CCC) is a network of blood vessels designed to preserve cerebral blood flow when primary routes fail [2]. Several recent studies have provided information about the role of collaterals in stroke pathophysiology, and it has been recognised that collateral circulation influences arterial recanalisation, reperfusion, haemorrhagic transformation and neurological outcomes after stroke [3-7]. To date, the CCC has mainly been assessed by using expensive or invasive imaging techniques.

Address for correspondence: Yuechun Li, Department of Neurology, Baotou Central Hospital, No.61 Huancheng Road, Donghe District, Baotou 014040, China, e-mail: liyuechum@163.com; Baojun Wang, Department of Neurology, Baotou Central Hospital, tel.: +86 04726955346

${ }^{*}$ Both authors contributed equally to this work 
The strength of the CCC varies among patients and partly depends on genetic and modifiable risk factors. Therefore, the identification of a biomarker of CCC status would be of considerable clinical significance.

MiRNAs are small non-coding RNAs ( 22 nucleotides) that suppress translation or induce degradation of downstream mRNA targets, thereby modulating gene expression at the post-transcriptional level [8]. Studies have shown that miRNAs play an important role in arteriogenesis and vascular remodelling $[9,10]$. Among the several miRNAs, miR-126 has appeared to be enriched in tissues with a high vascular component and to be specific to the vascular system $[11,12]$. miR-126, an endothelial cell-specific miRNA, modulates angiogenesis in vivo by enhancing the proangiogenic actions of VEGF, and repressing the expression of Spred-1 and PIK3R2 [13, 14]. It has been reported that there is a close relationship between miR-126 and coronary collateral vessel function, and these are considered as circulating biomarkers to identify insufficient or sufficient collateralisation in patients with chronic coronary occlusion $[15,16]$. Although miR-126 plays an important role in collateral circulation formation of the coronary artery, the correlation between miR-126 and the CCC has not been investigated.

In this single-centre cross-sectional study, we enrolled patients who underwent cerebral angiography with severe stenosis $(\geq 70 \%)$ or occlusion in at least one major intracranial artery. We aimed to investigate the correlation between the circulating miR-126 regulation pathway and the CCC, and to test whether miR-126 could serve as a potential biomarker for CCC formation.

\section{Materials and methods}

\section{Study design and population}

Inclusion criteria of this single-centre cross-sectional study were: (1) age 35-80 years; (2) hospitalised in the neurological department and having undergone cerebral angiography at Baotou Central Hospital; (3) having severe stenosis ( $\geq 70 \%)$ or occlusion in at least one major intracranial artery between December 2017 and July 2018.

Patients with any of the following factors, which were confirmed according to self-report, available medical records, and the judgement of physicians, were excluded: (1) acute cerebral infarction within the previous week; (2) various liver diseases and elevated transaminases (three times the normal level); (3) severe systemic organic disease (dysfunction of heart, lung, liver, kidney or other organs, and where survival time may be less than 12 months in the opinion of experienced physicians); (4) various tumours; (5) refusal to sign an informed consent form.

The CCC was graded using the American Society of Interventional and Therapeutic Neuroradiology/Society of Interventional Radiology (ASITN/SIR) Collateral Flow Grading System, which permitted dichotomisation into 'good' (grades 3-4) and 'poor' (grades 0-2) collateral flow: grade
0 (no collaterals visible at the ischaemic site); grade 1 (slow collaterals visible at the periphery of the ischaemic site with persistence of some of the defect); grade 2 (rapid collaterals at the periphery of the ischaemic site with persistence of some of the defect in only a portion of the ischaemic territory); grade 3 (collaterals with slow but complete angiographic blood flow of the ischaemic bed by the late venous phase); and grade 4 (complete and rapid collateral blood flow to the vascular bed in the entire ischaemic territory by retrograde perfusion).

We categorised ASITN/SIR into one of two classes (either poor or good) rather than the conventional three classes (poor, moderate or good) because of the small study sample and limited statistical power. Patients were divided into two groups according to their ASITN/SIR grade: patients with grade 3-4 (the good CCC group, $n=44$ ), and patients with grade $0-2$ (the poor CCC group, $\mathrm{n}=24$ ). Written consent was obtained from each subject, and the study protocol was approved by the Ethics Committee of Baotou Central Hospital.

\section{Determination of plasma miR-126}

Fasting blood samples $(5 \mathrm{~mL})$ were collected from the subjects via direct venous puncture, put into tubes containing sodium citrate, and then centrifuged at $2,000 \times \mathrm{g}$ for $10 \mathrm{~min}$. The supernatant (plasma) was then transferred carefully into tubes, which were stored in the refrigerator at $-80^{\circ} \mathrm{C}$. mRNAs were extracted from the plasma samples using the miRNeasy Plasma Kit (Qiagen). Both poly-(A) tailing and reverse transcription were performed on the samples with the miScript Reverse Transcription Kit (TIANGEN). miR-126 was quantified by quantitative reverse transcription polymerase chain reaction (qRT-PCR) assay, and U6 RNA was used as the miRNA internal control. Each reaction was performed using a miRNA-specific forward primer and a universal reverse primer according to the protocol of the manufacturer (TIANGEN). The primers were miR-126 forward: 5'-CGGGCCATTATTACTTTTG -3'; U6 forward: 5'-CTCGCTTCGGCAGCACA-3'. Each reaction was performed in a total volume of $20 \mathrm{uL}: 10.0 \mu \mathrm{L}$ TaqMan ${ }^{\circ}$ Fast Advanced Master Mix $(2 \times), 0.5 \mu \mathrm{L}$ miRNA-F $(10 \mu \mathrm{M})$, $0.5 \mu \mathrm{L}$ Universal miRNA-R $(10 \mu \mathrm{M}), 0.5 \mu \mathrm{L}$ Universal Taqman probe $(10 \mu \mathrm{M}), 1.0 \mu \mathrm{L}$ template DNA and nuclease-free $\mathrm{H}_{2} \mathrm{O}$ to adjust the volume. The PCR reaction was performed as follows: $50^{\circ} \mathrm{C}$ for $2.0 \mathrm{~min} ; 95^{\circ} \mathrm{C}$ for $20 \mathrm{sec}$, followed by 40 cycles $\left(95^{\circ} \mathrm{C}\right.$ for $5 \mathrm{sec} ; 60^{\circ} \mathrm{C}$ for $\left.25 \mathrm{sec}\right)$. Relative gene expression levels were analysed using the formula $2^{\Delta \mathrm{CT}}$, where $\Delta \mathrm{CT}=\mathrm{CT}$ (target gene) - CT (control), and this was repeated three times for each sample.

\section{Western blot method for Spred-1 and PIK3R2}

The whole blood total protein extraction kit (BB-3410, BestBio) was used to extract the total protein of the fasting blood sample. The bicinchoninic acid (BCA) quantitative kit was used to quantify the total protein. Protein concentrations were determined using a BCA protein assay kit (P0010, 
Beyotime). Each protein sample $(30 \mu \mathrm{g})$ was loaded for the assay. Nonspecific bands were blocked in TBS-T containing $5 \%$ non-fat milk for $1 \mathrm{~h}$ at room temperature. Membranes were subsequently incubated with the primary antibodies of rabbit anti-sprouty-related EVH1 domain containing protein 1 (SPRED-1) polyclonal antibody (1:500; ab64740, Abcam), rabbit anti-phosphoinositol-3 kinase regulatory subunit 2 (PIK3R2) polyclonal antibody (1:1,000; PA5-15268, Thermo Fisher), followed by goat anti-mouse $\operatorname{IgG}(\mathrm{H}+\mathrm{L})$ secondary antibody $(1: 5,000 ; 31160$, Thermo Pierce) and goat anti-rabbit IgG $(\mathrm{H}+\mathrm{L})$ secondary antibody $(1: 5,000 ; 31210$, Thermo Pierce) for $1 \mathrm{~h}$ at room temperature. The immunocomplexes were visualised by SuperSignal ${ }^{\circ}$ West Dura Extended Duration Substrate. The film signals were digitally scanned and then quantified using BandScan5.0 software (Bio-Rad, USA). An antibody for transferrin (1:2,000; MAB5746, R\&D Systems) was used as an internal control. Data was provided in terms of the mean \pm standard deviation of the percentage ratio of the control. Each band was repeated three times, and the relative expression of target protein was expressed as \{target protein/ /internal parameters (optical density value) $\} \times 10^{\mathrm{n}}$. Results are expressed as mean \pm standard deviation.

\section{ELISA assay for plasma VEGF levels}

All fasting blood samples ( $5 \mathrm{~mL}$ per patient) were collected via a direct venous puncture, placed into tubes containing sodium citrate, and then centrifuged at 2,000 $\times \mathrm{g}$ for $10 \mathrm{~min}$. The layer of the supernatant (plasma) was carefully transferred into other tubes and stored at $-20^{\circ} \mathrm{C}$. We used the Human VEGF ELISA Kit (R\&D Systems) to measure the plasma levels of VEGF, following the manufacturer's instructions. Absorbance was measured at $450 \mathrm{~nm}$ (primary wavelength).

\section{Other variables of interest}

Several clinical characteristics were compared between the groups:

(1) demographics (age and sex) and medical histories, including hypertension (according to self-report, available medical records, taking hypotensive medication, systolic blood pressure $\geq 140 \mathrm{mmHg}$ on a different day, or diastolic blood pressure $\geq 90 \mathrm{mmHg}$ on a different day), diabetes (according to self-report, available medical records, taking antidiabetic medication, or fasting blood glucose $\geq 7.0 \mathrm{mmol} / \mathrm{L}$ ), coronary heart disease (diagnosed by self-report or available medical records), and hyperlipidemia (diagnosed according to in-hospital tests or medical records, meeting at least one of the following criteria: blood cholesterol $\geq 6.2 \mathrm{mmol} / \mathrm{L}$; LDL-C $\geq 4.1 \mathrm{mmol} / \mathrm{L}$; blood triglyceride $\geq 2.3 \mathrm{mmol} / \mathrm{L}$; or HDL-C $<1.0 \mathrm{mmol} / \mathrm{L}$ );

(2) BMI $\left(\mathrm{kg} / \mathrm{m}^{2}\right)$; smoking, defined as regularly smoking (smoking $\geq 1$ cigarette/day on average for longer than six months) or occasionally smoking (smoking more than four times a week and but less than one cigarette/day on average); drinking, defined as regular drinking (drinking $\geq 1$ time/ /week, drinking $\geq 50 \mathrm{ml}$ alcohol each time, for more than half a year), or occasional drinking (drinking 1-3 times/month, drinking $\geq 50 \mathrm{ml}$ alcohol each time, for more than half a year);

(3) fasting blood sample tests, including cholesterol, triglyceride, LDL-C, HDL-C, glucose, creatinine, and homocysteine;

(4) medication histories, including aspirin, clopidogrel, ACEI/ /ARBs, statins, CCB, and beta-blockers.

\section{Statistical analyses}

The continuous variables are expressed as mean \pm standard deviation, and the categorical variables are expressed as frequencies. The Kolmogorov-Smirnov test was used to test whether the data were normally distributed. The differences in continuous clinical characteristics between the groups were assessed using the Student t-test or Mann-Whitney U test. Associations between categorical variables were tested by Pearson's $\chi 2$ test. Associations of miR-126, Spred-1, PIK3R2, VEGF-A with collateral grade were assessed using the Spearman correlation test. Associations of miR-126 with VEGF, Spred-1 and PIK3R2 were also analysed with Spearman correlation test because: (1) data on miR-126, Spred-1, and PIK3R2 do not have a normal distribution; (2) to keep consistency with the previous analysis (association of miR-126 and other markers with CCC status).

Receiver operating characteristic (ROC) curves were established to evaluate the predictive power of circulating miR-126 for the CCC status of patients. The area under the curve (AUC) was used to assess the predictive power. We only performed univariate analysis for three reasons: firstly, patients with severe organ dysfunction, liver diseases, tumours and other diseases which might affect circulating biomarkers of interest were excluded from the study; secondly, the baseline characteristics, including demographic factors, medical histories and laboratory tests which might have an influence on miR-126 level were generally comparable between the good CC group and the poor CCC group; and thirdly, the small study sample further restrained our statistical power to perform multivariate analysis. Statistical analyses were conducted using IBM SPSS Statistics 19, and $\mathrm{p}<0.05$ was considered statistically significant.

\section{Results}

\section{Baseline characteristics}

Of 80 patients examined for eligibility, 10 were excluded (eight with acute cerebral infarction, one with tumour, and one with severe systemic organ disease). Of the 70 patients confirmed eligible, two were excluded due to refusing consent.

So, in total 68 patients were included in this study, 44 with good CCC and 24 with poor CCC. All enrolled patients were found $>70 \%$ stenosis or occlusion, via TCD, CTA, or MRA, 
Table 1. Clinical characteristics and biochemical parameters of patients

\begin{tabular}{|c|c|c|c|}
\hline \multirow[t]{2}{*}{ Variables } & \multicolumn{2}{|c|}{ ASITN/SIR grade } & \multirow[t]{2}{*}{ P-value } \\
\hline & Good CCC $(n=44)$ & Poor CCC $(n=24)$ & \\
\hline \multicolumn{4}{|l|}{ Clinical characteristics } \\
\hline Age (y) & $59.52 \pm 9.45$ & $62.29 \pm 8.75$ & 0.240 \\
\hline Male n (\%) & $35(79.5 \%)$ & $18(75 \%)$ & 0.666 \\
\hline $\mathrm{BMI}(\mathrm{kg} / \mathrm{m} 2)$ & $25.39 \pm 3.21$ & $25.54 \pm 3.24$ & 0.850 \\
\hline Hypertension n (\%) & $30(68.2 \%)$ & $20(83.3 \%)$ & 0.176 \\
\hline Diabetes $n(\%)$ & $11(25 \%)$ & $10(41.7 \%)$ & 0.155 \\
\hline Coronary heart disease $\mathrm{n}(\%)$ & $10(22.7 \%)$ & $4(16.7 \%)$ & 0.555 \\
\hline Smoking n (\%) & $26(59.1 \%)$ & $13(54.2 \%)$ & 0.695 \\
\hline Drinking n (\%) & $16(36.4 \%)$ & $11(45.8 \%)$ & 0.446 \\
\hline Hyperlipemia n (\%) & $18(40.9 \%)$ & $6(25 \%)$ & 0.190 \\
\hline \multicolumn{4}{|l|}{ Laboratory index } \\
\hline $\mathrm{TC}(\mathrm{mmol} / \mathrm{l})$ & $4.03 \pm 0.93$ & $4.15 \pm 1.14$ & 0.635 \\
\hline $\mathrm{TG}(\mathrm{mmol} / \mathrm{l})$ & $1.84 \pm 1.11$ & $1.60 \pm 0.68$ & 0.691 \\
\hline LDL-C (mmol/l) & $2.34 \pm 0.71$ & $2.29 \pm 1.02$ & 0.836 \\
\hline HDL-C (mmol/l) & $0.98 \pm 0.21$ & $1.10 \pm 0.44$ & 0.273 \\
\hline $\mathrm{Glu}(\mathrm{mmol} / \mathrm{l})$ & $5.98 \pm 2.02$ & $6.08 \pm 1.77$ & 0.657 \\
\hline Creatinine $(\mu \mathrm{mol} / \mathrm{l})$ & $66.23 \pm 13.64$ & $60.08 \pm 12.92$ & 0.075 \\
\hline Homocysteine(umol/l) & $14.56 \pm 8.99$ & $16.81 \pm 10.33$ & 0.229 \\
\hline \multicolumn{4}{|l|}{ Medication history } \\
\hline Aspirin n (\%) & $44(100 \%)$ & $24(100 \%)$ & NA \\
\hline Clopidogrel n (\%) & $38(86.4 \%)$ & $24(100 \%)$ & 0.083 \\
\hline ACEI/ARBs $n(\%)$ & $9(20.5 \%)$ & $4(16.7 \%)$ & 1.000 \\
\hline Statin n (\%) & $44(100 \%)$ & $24(100 \%)$ & NA \\
\hline CCB n (\%) & $8(18.2 \%)$ & $0(0 \%)$ & 0.043 \\
\hline Beta-blocker n (\%) & $8(18.2 \%)$ & $7(29.2 \%)$ & 0.364 \\
\hline
\end{tabular}

in at least one major intracranial artery, and were advised to perform digital subtraction angiography (DSA) to verify if interventional therapy was justified. The distribution of cerebral vascular stenosis/occlusion among all patients in both groups are shown in the supplement. Their clinical characteristics, biochemical parameters and medication history, including age, BMI, sex, rates of hypertension, coronary heart disease, diabetes mellitus and hyperlipemia, smoking history, drinking history, fasting glucose, and lipid profiles including LDL, HDL, TG, TC, homocysteine and creatinine, are set out in Table 1. The number of patients who were taking aspirin, clopidogrel, ACEIs/ARBs, statins and beta-blockers did not differ between the two groups. The difference in the calcium antagonist of medication history between the two groups was statistically significant $(\mathrm{p}<0.05)$.

Levels of plasma miR-126, VEGF, Spred-1 and PIK3R2 in patients classified by CCC status
The concentrations of plasma VEGF-A in the good CCC group and the poor CCC group were $103.66 \pm 8.00 \mathrm{pg} / \mathrm{ml}$ and $75.82 \pm 15.69 \mathrm{pg} / \mathrm{ml}$, respectively. Compared to the poor CCC group, the plasma level of VEGF was significantly higher in the good CCC group ( $<$ 0.05) (Fig. 1A).

Subsequently, we tested the levels of plasma miR-126, Spred-1 and PIK3R2 in patients with intracranial artery occlusion or stenosis of $\geq 70 \%$ according to their CCC status. Results showed that the level of plasma miR-126 was 1.81-fold higher in the good CCC group $(14.76 \pm 11.91 \mathrm{pg} / \mathrm{ml})$ than in the poor CCC group $(8.17 \pm 4.45 \mathrm{pg} / \mathrm{ml}, \mathrm{p}<0.05)$ (Fig. 1B). Moreover, the protein level of Spred-1 and PIK3R2 in plasma was lower in the good CCC group than in the poor CCC group (Spred-1:0.23 $\pm 0.09 \mathrm{pg} / \mathrm{ml}$ for good CCC group, $0.83 \pm 0.12 \mathrm{pg} / \mathrm{ml}$ for poor CCC group; PIK3R2: $0.22 \pm 0.15 \mathrm{pg} / \mathrm{ml}$ for good CCC group, $0.65 \pm 0.23 \mathrm{pg} / \mathrm{ml}$ for poor CCC group) (Fig. 2). 


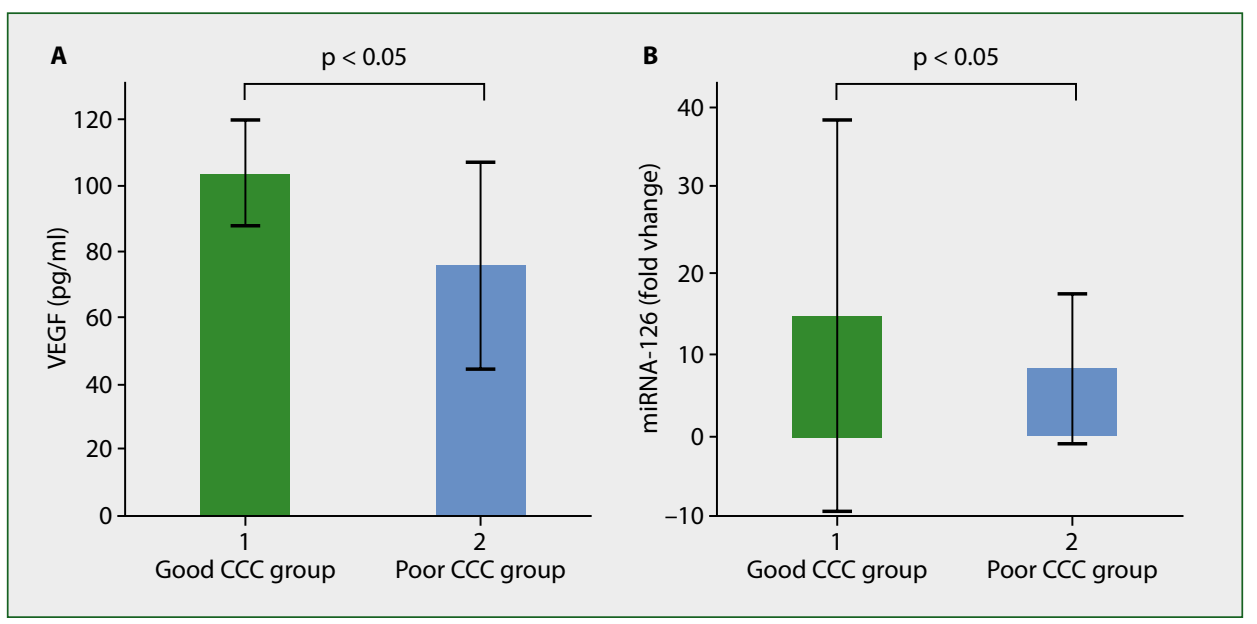

Figure 1. Comparison of plasma miR-126 and VEGF levels between good and poor CCC groups. A. Plasma level of VEGF in patients; B. Plasma level of miR-126 in patients

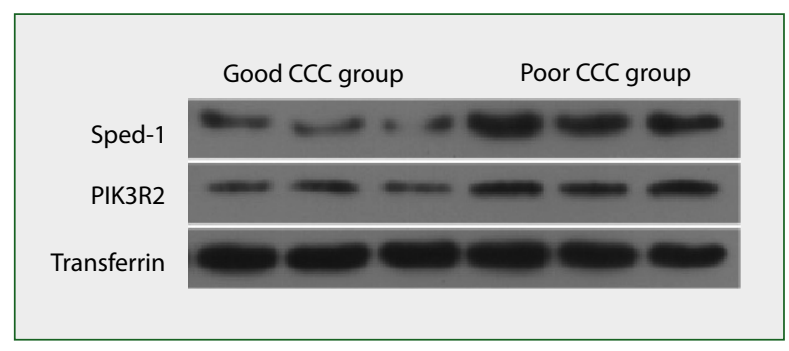

Figure 2. Protein level of Spred-1 and PIK3R2 correlation between miR126 and VEGF and ASITN/SIR score $(\mathrm{R}=0.595, \mathrm{p}<0.01)$. Conversely, ASITN/SIR score was negatively correlated with Spred-1 $(\mathrm{R}=-0.817, \mathrm{p}<0.01)$ and PIK3R2 (R = -0.513, $\mathrm{p}=0.01)$ (Fig. 4).

\section{Correlation between miR-126 and VEGF, Spred-1 and PIK3R2}

To explore the mechanism of miR-126 in improving collateral circulation, we analysed the correlation

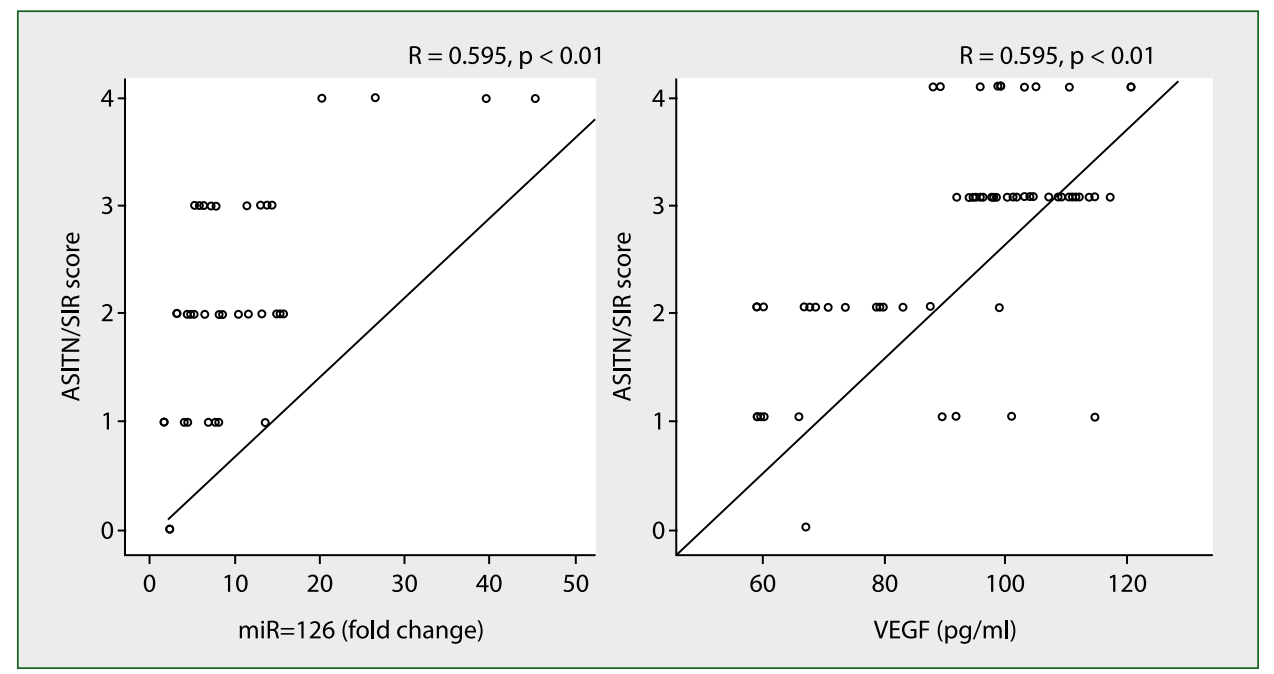

Figure 3. Correlation between miR-126 and VEGF and ASITN/SIR score

Correlation of miR-126, VEGF, Spred-1 and PIK3R2 with ASITN/SIR score

We further investigated the correlation of miR-126 and VEGF with ASITN/SIR score, which was used to evaluate the degree of CCC. As shown in Figure 3, there was a positive between miR-126 and VEGF, Spred-1 and PIK3R2. There was a positive correlation between miR-126 and VEGF $(\mathrm{R}=0.333, \mathrm{p}=0.041$ ) (Fig. 5). However, the correlation between plasma miR-126 and Spred-1 and PIK3R2 was not significant (Fig. 6, 7). 


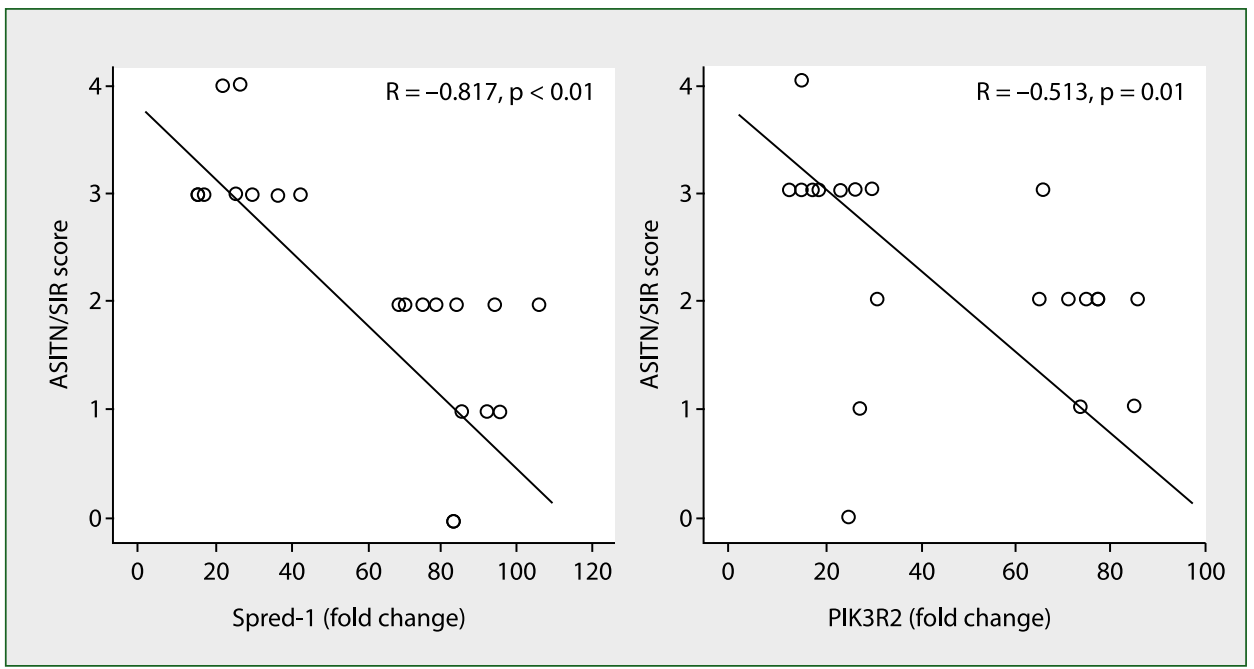

Figure 4. Correlation between Spred-1 and PIK3R2 and ASITN/SIR score

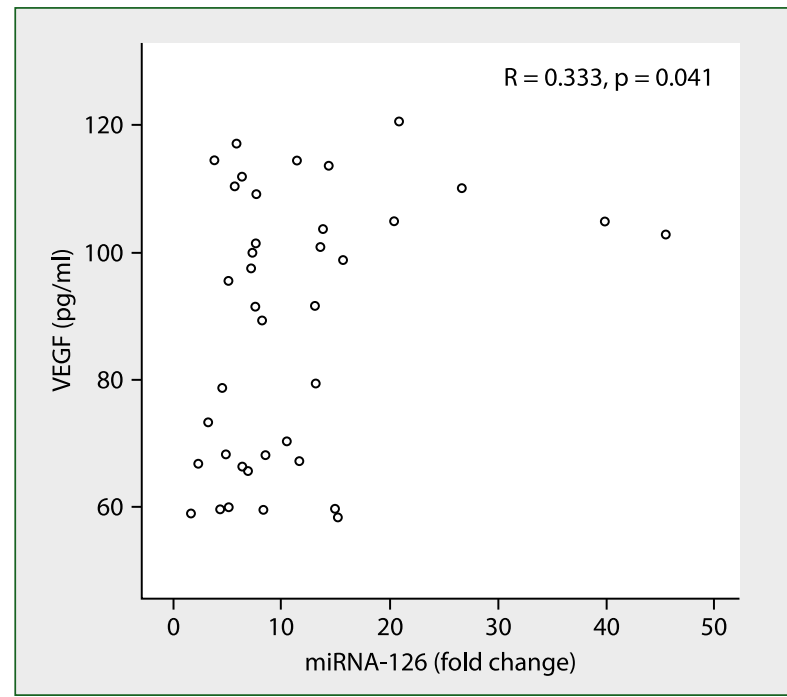

Figure 5. Correlation between plasma miR-126 and VEGF

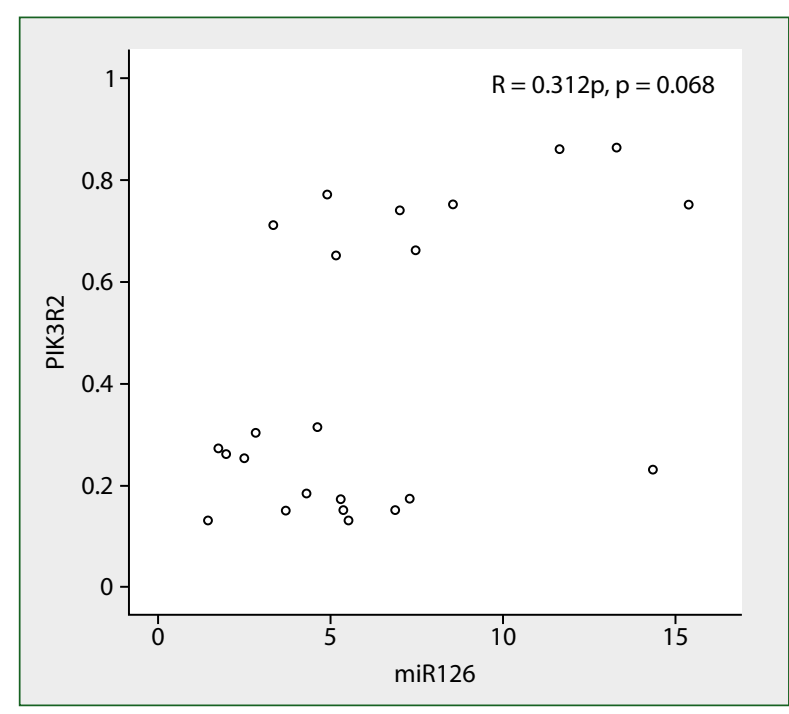

Figure 7. Correlation of plasma miR-126 with PIK3R2

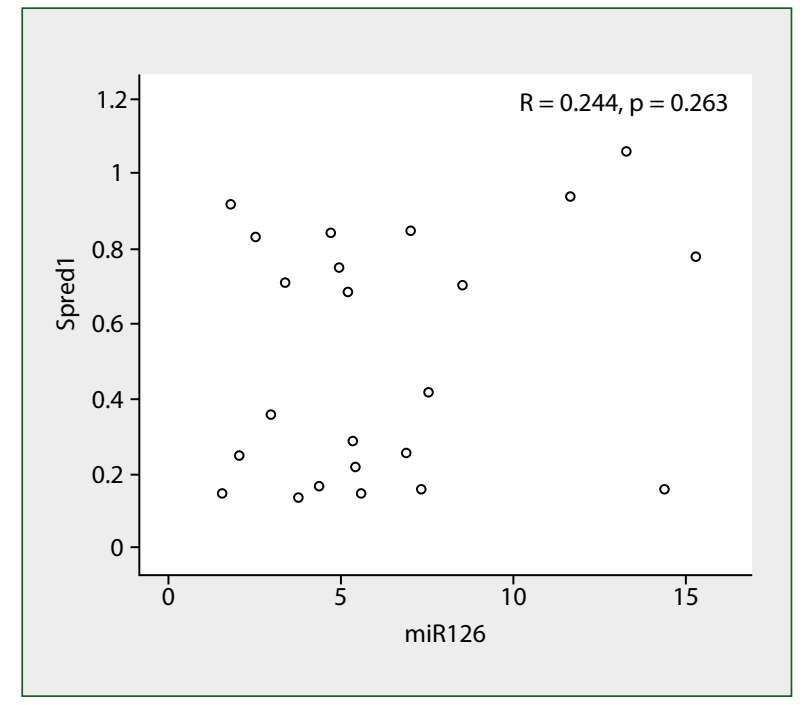

Figure 6. Correlation of plasma miR-126 with Spred1

Comparison of predictive power of miR-126 for CCC status

To compare the predictive power of plasma miR-126 for CCC status, we performed ROC analysis for the patients. As shown in Figure 8, the AUC for miR-126 was 0.328 (95\% CI: 0.158-0.498; $\mathrm{p}=0.067)$.

\section{Discussion}

In this study, we found that plasma miR-126 and VEGF were significantly higher in patients in the good CCC group than in those in the poor CCC group. In contrast, plasma Spred-1 and PIK3R2 levels were lower in patients in the good CCC group. In addition, miR-126 and VEGF were positively correlated with ASITN/SIR, whereas Spred-1 and PIK3R2 were negatively correlated with ASITN/SIR. However, miR-126 might not be an independent predictor for CCC, as the AUC of the marker for CCC status was only 0.328 (95\% CI: 0.158-0.498; p = 0.067). 


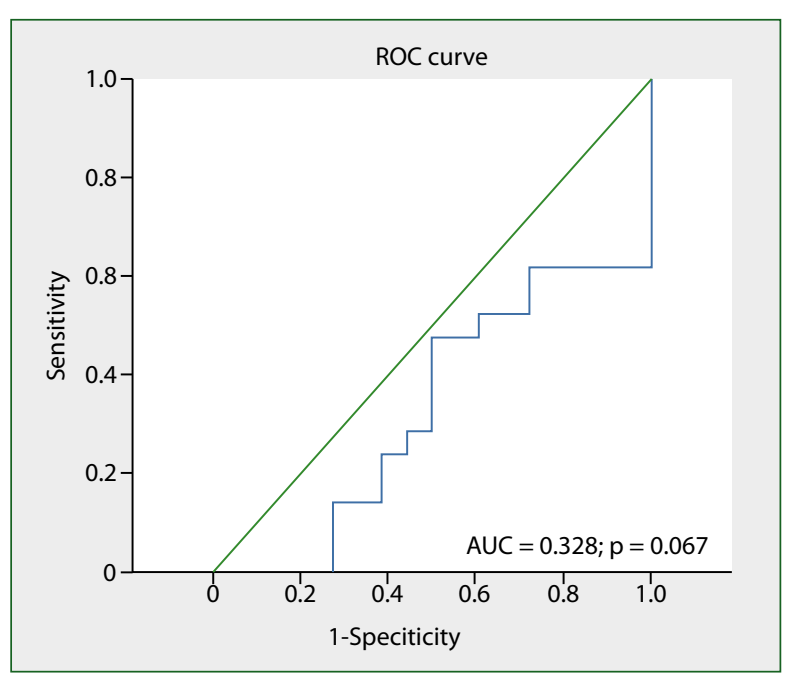

Figure 8. Predictive power of miR-126 for CCC

miR-126, the angiogenesis-related miRNA, is regarded as one of the main regulators of physiological angiogenesis. [13, 17, 18]. Several recent studies have identified that miR-126 promotes VEGF expression by suppressing Spred-1 and PIK3R2 expression [14], which contributes to angiogenesis [19-22]. Nie et al. [23] reported that plasma miR-126 level is positively correlated with CCC formation and is an independent predictor of its development in patients with severely narrowed coronary arteries. To date, it has not been reported whether miR-126 can be used as a biomarker for cerebrovascular collateral circulation. We first found that plasma miR-126 and VEGF were significantly higher in patients in the good CCC group than those in the poor CCC group among patients with at least one major intracranial artery severe stenosis $(\geq 70 \%)$ or occlusion. Plasma miR-126 and VEGF were positively correlated with ASITN/SIR, whereas Spred-1 and PIK3R2 were negatively correlated with ASITN/SIR. Although our ROC analysis found insignificant AUC value, the abovementioned findings still support our hypothesis that higher miR-126 was associated with better CCC status, possibly mediated by increasing VEGF and reducing Spred-1 and PIK3R2 levels.

Our study has several limitations. Firstly, we did not analyse the effects of other factors on miR-126, for which the reasons are mentioned above (in the Statistical Analysis). It is known that hypoxia can induce miR-126 expression [24]. So, it is possible that more severe stenosis of cerebral arteries promotes better CCC status in the good CCC group, and simultaneously increased the miR-126 level. But the severity of stenosis was generally comparable between the groups in our study (see Supplement). Moreover, it has also been reported that proatherogenic metabolic factors, such as diabetes and hyperlipidemia, might down-regulate miR-126 expression [24].

Therefore, association between miR-126 and collateral flow might be complicated by arterial stenosis and metabolic disorders. The relationship between miR-126 level, metabolic risk factors, cerebral artery stenosis and collateral circulation remains to be elucidated.

Secondly, the small sample size in our study restrained statistical power to fully evaluate association of miR- 126 with CCC status. In combination with the single-centre cross-sectional design and male-predominant population (more than $70 \%$ ), our findings might not be fully generalisable to other studies.

Finally, this is an exploratory study that lacks evidence of repeated comparisons, and therefore further research should be conducted to confirm the results.

\section{Conclusions}

Plasma miR-126 level may be related to better CCC formation accompanied by upregulation of VEGF and reduced Spred-1 and PIK3R2 protein expression. However, miR-126 might not be an independent predictor for CCC, given its low predictive value.

Funding: This work was funded by the project of the Natural Science Foundation of Inner Mongolia Autonomous Region (No. 2017MS0805).

\section{References:}

1. Goyal M, Demchuk AM, Menon BK, et al. ESCAPE Trial Investigators. Randomized assessment of rapid endovascular treatment of ischemic stroke. N Engl J Med. 2015; 372(11): 1019-1030, doi: 10.1056/ NEJMoa1414905, indexed in Pubmed: 25671798.

2. Sheth SA, Liebeskind DS. "Imaging Evaluation of Collaterals in the Brain: Physiology and Clinical Translation”. Curr Radiol Rep. 2014; 2(1): 29, doi: 10.1007/s40134-013-0029-5, indexed in Pubmed: 25478305.

3. Villringer K, Zimny S, Galinovic I, et al. The Association Between Recanalization, Collateral Flow, and Reperfusion in Acute Stroke Patients: A Dynamic Susceptibility Contrast MRI Study. Front Neurol. 2019; 10: 1147, doi: 10.3389/fneur.2019.01147, indexed in Pubmed: 31708866.

4. Shah VS, Eaton RG, Cua S, et al. Scoring of Middle Cerebral Artery Collaterals Predicts RAPID CT-Perfusion Analysis and Short-Term Outcomes in Acute Ischemic Stroke Patients Undergoing Thrombectomy. World Neurosurg. 2020; 135: e494-e499, doi: 10.1016/j. wneu.2019.12.033, indexed in Pubmed: 31843729.

5. Leng X, Fang H, Leung TWH, et al. Impact of collaterals on the efficacy and safety of endovascular treatment in acute ischaemic stroke: a systematic review and meta-analysis. J Neurol Neurosurg Psychiatry. 2016; 87(5): 537-544, doi: 10.1136/jnnp-2015-310965, indexed in Pubmed: 26063928.

6. Liebeskind DS, Tomsick TA, Foster LD, et al. IMS III Investigators. Collaterals at angiography and outcomes in the Interventional Management of Stroke (IMS) III trial. Stroke. 2014; 45(3): 759-764, doi: 10.1161/STROKEAHA.113.004072, indexed in Pubmed: 24473178.

7. Guglielmi V, LeCouffe NE, Zinkstok SM, et al. MR-CLEAN Registry Investigators. Collateral Circulation and Outcome in Atherosclerotic Versus Cardioembolic Cerebral Large Vessel Occlusion. Stroke. 2019; 50(12): 3360-3368, doi: 10.1161/STROKEAHA.119.026299, indexed in Pubmed: 31658903. 
8. Huntzinger $E$, Izaurralde $E$. Gene silencing by microRNAs: contributions of translational repression and mRNA decay. Nat Rev Genet. 2011; 12(2): 99-110, doi: 10.1038/nrg2936, indexed in Pubmed: 21245828.

9. Nazari-Jahantigh $\mathrm{M}$, Wei $\mathrm{Y}$, Schober $\mathrm{A}$. The role of microRNAs in arterial remodelling. Thromb Haemost. 2012; 107(4): 611-618, doi: 10.1160/TH11-12-0826, indexed in Pubmed: 22371089.

10. Welten SMJ, Bastiaansen AJ, de Jong RCM, et al. Inhibition of $14 q 32$ MicroRNAs miR-329, miR-487b, miR-494, and miR-495 increases neovascularization and blood flow recovery after ischemia. Circ Res. 2014; 115(8): 696-708, doi: 10.1161/CIRCRESAHA.114.304747, indexed in Pubmed: 25085941.

11. Lagos-Quintana M, Rauhut R, Yalcin A, et al. Identification of tissue-specific microRNAs from mouse. Curr Biol. 2002; 12(9): 735 739, doi: 10.1016/s0960-9822(02)00809-6, indexed in Pubmed: 12007417.

12. Wienholds E, Kloosterman WP, Miska E, et al. MicroRNA expression in zebrafish embryonic development. Science. 2005; 309(5732): 310311, doi: 10.1126/science.1114519, indexed in Pubmed: 15919954.

13. Wang S, Aurora AB, Johnson BA, et al. The endothelial-specific microRNA miR-126 governs vascular integrity and angiogenesis. Dev Cell. 2008; 15(2): 261-271, doi: 10.1016/j.devcel.2008.07.002, indexed in Pubmed: 18694565.

14. Fish JE, Santoro MM, Morton SU, et al. miR-126 regulates angiogenic signaling and vascular integrity. Dev Cell. 2008; 15(2): 272-284, doi: 10.1016/j.devcel.2008.07.008, indexed in Pubmed: 18694566.

15. Hakimzadeh N, Nossent AY, van der Laan AM, et al. Circulating MicroRNAs Characterizing Patients with Insufficient Coronary Collateral Artery Function. PLoS One. 2015; 10(9): e0137035, doi: 10.1371/journal. pone.0137035, indexed in Pubmed: 26331273.

16. Nie X, Su L, Zhou Y, et al. [Association between plasma levels of microRNA-126 and coronary collaterals in patients with coronary artery disease]. Zhonghua Xin Xue Guan Bing Za Zhi. 2014; 42(7): 561-565, indexed in Pubmed: 25327597.
17. Huang $F$, Zhu $X$, Hu XQ, et al. Mesenchymal stem cells modified with miR-126 release angiogenic factors and activate Notch ligand Delta-like-4, enhancing ischemic angiogenesis and cell survival. Int J Mol Med. 2013; 31(2): 484-492, doi: 10.3892/ijmm.2012.1200, indexed in Pubmed: 23229021.

18. Hu J, Zeng L, Huang J, et al. miR-126 promotes angiogenesis and attenuates inflammation after contusion spinal cord injury in rats. Brain Res. 2015; 1608: 191-202, doi: 10.1016/j.brainres.2015.02.036, indexed in Pubmed: 25724143.

19. Ye L, Peng Y, Mo J, et al. MiR-126 enhances VEGF expression in induced pluripotent stem cell-derived retinal neural stem cells by targeting spred-1. Int J Clin Exp Pathol. 2018; 11(2): 1023-1030, indexed in Pubmed: 31938197.

20. Meng S, Cao JT, Zhang B, et al. Downregulation of microRNA-126 in endothelial progenitor cells from diabetes patients, impairs their functional properties, via target gene Spred-1. J Mol Cell Cardiol. 2012; 53(1): 64-72, doi: 10.1016/j.yjmcc.2012.04.003, indexed in Pubmed: 22525256.

21. Ji JS, Xu M, Song JJ, et al. Inhibition of microRNA-126 promotes the expression of Spred1 to inhibit angiogenesis in hepatocellular carcinoma after transcatheter arterial chemoembolization: in vivo study. Onco Targets Ther. 2016; 9: 4357-4367, doi: 10.2147/0TT.S106513, indexed in Pubmed: 27499630.

22. Wang L, Lee AY, Wigg JP, et al. miR-126 Regulation of Angiogenesis in Age-Related Macular Degeneration in CNV Mouse Model. Int J Mol Sci. 2016; 17(6), doi: 10.3390/ijms17060895, indexed in Pubmed: 27338342.

23. Nie X, Su L, Zhou Y, et al. Association between plasma levels of microRNA-126 and coronary collaterals in patients with coronary artery disease. Zhonghua Xin Xue Guan Bing Za Zhi. 2014; 42(7): 561-565, indexed in Pubmed: 25327597.

24. Chistiakov DA, Orekhov AN, Bobryshev YV. The role of miR-126 in embryonic angiogenesis, adult vascular homeostasis, and vascular repair and its alterations in atherosclerotic disease. J Mol Cell Cardiol. 2016; 97: 47-55, doi: 10.1016/j.yjmcc.2016.05.007, indexed in Pubmed: 27180261. 